\title{
Variation of proton radiation belt deduced from solar cell degradation of Akebono satellite
}

\author{
Hiroyuki Ishikawa $^{1}$, Wataru Miyake ${ }^{1}$, and Ayako Matsuoka ${ }^{2}$ \\ ${ }^{1}$ Department of Aeronautics and Astronautics, Tokai University, Japan \\ ${ }^{2}$ Institute of Space and Astronautical Science, Japan
}

(Received February 16, 2012; Revised May 15, 2012; Accepted June 14, 2012; Online published March 6, 2013)

\begin{abstract}
We have analyzed the degradation of the solar cells of the Akebono satellite due to various space radiations, and found a signature of a deformed proton radiation belt in 1991. The output current of the solar cells decreased slowly from 13A in 1989 to about 7A in 2009, with a quasi-periodic oscillation. By comparing the decrease rate with the solar proton flux measured by GOES satellites and the flux of trapped radiations from NASA's models (AP8MAX and AE8MAX), we found a fair correlation between the decrease rate of the solar cell output current and the trapped proton flux between 1989 and 1992. However, we also found an interval of inconsistency after a large solar proton event and geomagnetic storm in March 1991. Modified models of trapped proton flux distribution with a second peak around $L=2.3$ lead to a better coincidence during the interval, indicating that the deformation of the proton radiation belt terminated around the end of 1991 or early 1992. The result provides further evidence for the proton radiation belt, usually thought as being quite stable, changing drastically on special occasions.
\end{abstract}

Key words: Proton radiation belt, solar cell degradation, Akebono satellite.

\section{Introduction}

A solar active region produced a X9/3B flare at 2247 UT on 22 March, 1991, and a M6/2B flare at 0429 UT on 23 March, 1991. Severe geomagnetic storm conditions followed the arrival of an interplanetary shock at 0342 UT on 24 March, 1991. A second shock arrival was observed at 1930 UT on 24 March, 1991, after which the magnetopause was compressed to within the GOES satellite geosynchronous orbit. The GOES satellite observed an associated proton event at greater than $10 \mathrm{MeV}$ with a peak flux of 43,000 pfu at 0350 UT on 24 March, 1991, coinciding with the first shock arrival.

The harsh space events drastically changed the terrestrial radiation belt. Blake et al. (1992) reported that the CRRES satellite detected a sudden appearance of protons and electrons with energies of a few tens of $\mathrm{MeV}$ around $L=2.6$, just after the first shock arrival. The energetic particles were presumably injected by the interaction between the shock and the terrestrial magnetosphere, and formed a new radiation belt between $L=2$ and $L=3.5$. The formation process of the new radiation belt has been a subject of several studies (e.g., Li et al., 1993; Hudson et al., 1995, 1997; Obara and Li, 2003). The new radiation belt lasted for months with a slow variation and decay, at least until the CRRES satellite failed in mid October 1991 (e.g., Gussenhoven et al., 1996).

A remnant of the new electron radiation belt was still

Copyright (C) The Society of Geomagnetism and Earth, Planetary and Space Sciences (SGEPSS); The Seismological Society of Japan; The Volcanological Society of Japan; The Geodetic Society of Japan; The Japanese Society for Planetary Sciences; TERRAPUB.

doi:10.5047/eps.2012.06.004 found by the SAMPEX satellite, launched on 3 July, 1992 (Looper et al., 1994; Li and Temerin, 2001), which slowly decayed and diffused inward. The proton radiation belt, on the other hand, seemed to have already lost the newlyformed secondary peak around $L=2 \sim 3$ when the SAMPEX satellite started its observation in 1992 (Looper $e t$ al., 2005). Because of lack of any other proton observation in the inner magnetosphere, we do not know how the proton radiation belt returned to the usual state.

The Japanese Akebono satellite stayed deep in the inner magnetosphere and orbited in the radiation belt environment covering the whole interval of interest in 1991 and 1992. Whereas the SAMPEX satellite observed the proton radiation belt only at a low altitude of a $550 \times 675 \mathrm{~km}$ orbit, the higher apogee of the Akebono satellite allowed it to enter the midst of the proton radiation belt. Solar cells on board any satellites gradually degrade in the harsh environment of various space radiations during years in flight. In this paper, we present a signature of the deformed proton radiation belt in 1991 by analyzing the variation of the solar cell output current of the Akebono satellite.

\section{Data}

The Akebono satellite was launched on 22 February, 1989 , into an elliptical orbit with an inclination of $75.1^{\circ}$ and with an initial apogee and perigee of 10,482 and $272 \mathrm{~km}$, respectively. The satellite is dedicated to the study on the auroral particle acceleration and related phenomena over the polar region. Details of the satellite are described elsewhere (Oya and Tsuruda, 1990; Tsuruda and Oya, 1991). The satellite has been successfully operated over more than 20 years and a huge data base has been built. 


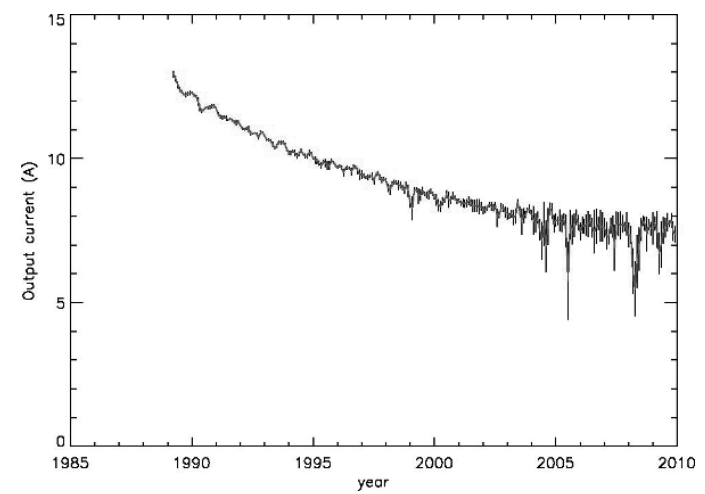

Fig. 1. Monthly-average with RMS (root mean square) bars of the solar cell output current of the Akebono satellite under sunlit conditions from 1989 up to the end of 2009.

Figure 1 shows the variation of the monthly-averaged solar cell output current of the Akebono satellite under sunlit conditions from 1989 through 2009. Black vertical bars indicate the RMS (root mean square) variation around a monthly average. We have already removed the annual oscillation component associated with the variation of the heliocentric distance of the earth orbit. The output is continuously decreased over 20 years. Besides the continuous decrease, short-term, sometimes irregular, variations are overlaid, especially in the later interval. RMS for a monthlyaverage is also consistently larger in the later interval.

The long-term continuous decrease throughout the entire period is probably due to the total dose effect of the space radiation environment. The decrease was initially fast, but it slowed down in later years, so that it is close to an exponential variation under a constant radiation (e.g., Patel, 2005). The short-term irregular variations, on the other hand, are not thought to be attributed to the space radiation effect, since they are too large and too abrupt.

One possible cause is the temperature variation after the degradation of solar cells reached quite a significant level. The output current is decreased at high temperature (e.g., Patel, 2005). The satellite power system is initially designed to provide a stable output level with enough margin for the expected temperature range. The margin became smaller with the progress of degradation. The output current became more susceptible to temperature variation, and occasionally decreased under a high temperature condition. This high temperature is caused by the Earth's albedo when the satellite is located at a low altitude over dayside.

Another cause is a quite small amount of Akebono data in the later years, which eventually enhances the temperature effect. In later years, the Akebono data were acquired during a quite limited time in a month. It can happen that most of the data in a certain month are obtained when the satellite is under a high-temperature condition, and monthly averaging cannot work for minimizing the temperature effect. We have actually confirmed a relation between high temperature and the decrease of output current for the Akebono data in the later years. We do not show the results here, but will report them in a separate paper.

In this study, we analyze the current output data of the Akebono solar cells before 1993. Since the total degrada-
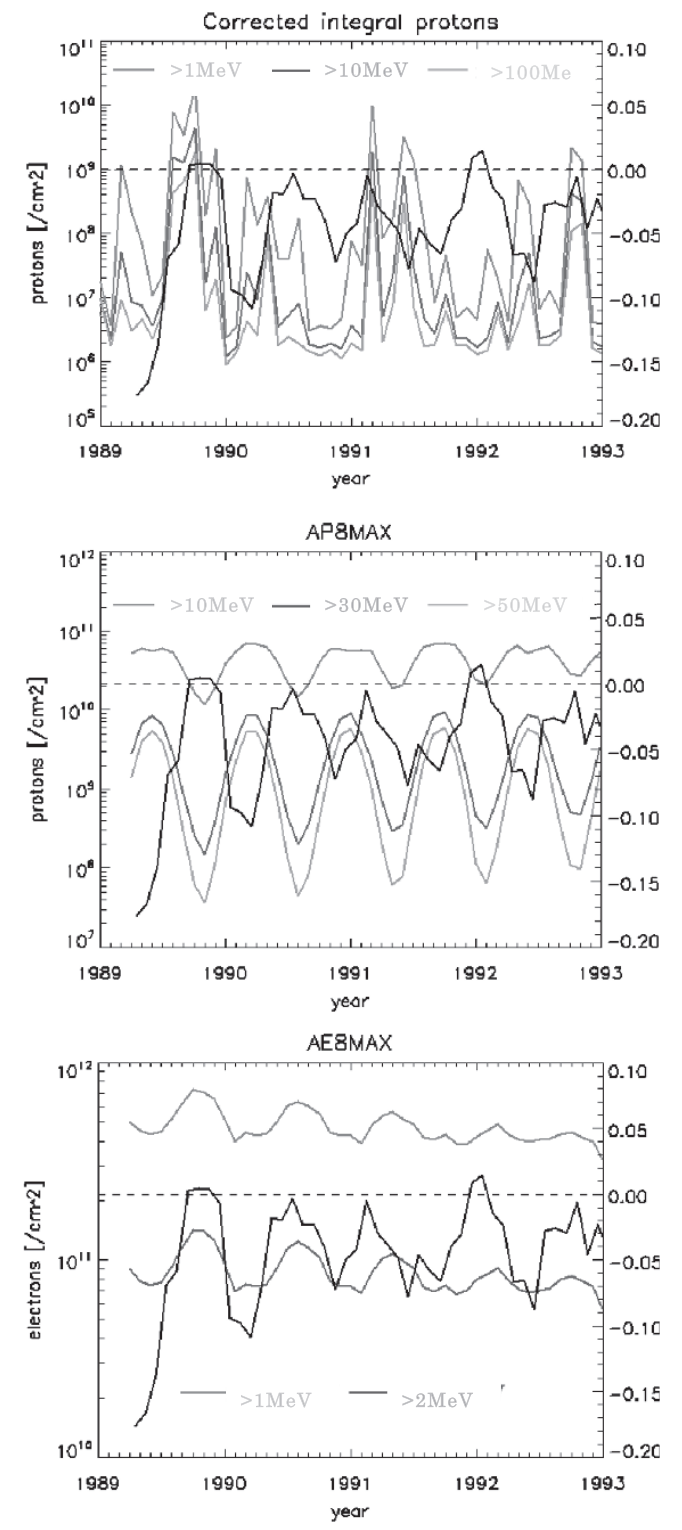

Fig. 2. Comparison between the variance of the solar cell output current per month (black lines) and solar proton flux (top panel), trapped proton flux (middle), and trapped electron flux (bottom), integrated over a month. Colors of the particle flux line represent the energy range as indicated in the figure.

tion was still small and the temperature effect was not so significant before 1993, we expect that space radiation is most responsible for the variation of the current output for the interval. We can confirm that the RMS variation around a monthly average is small before 1993 in Fig. 1 and we simply assume that we can derive the space radiation effect by using the monthly average of the output current. We also use the solar proton data from GOES measurements in GEO and trapped particle models of NASA's AP8MAX and AE8MAX, and investigate any relationship between the decrease of output current and energetic particle fluxes.

\section{Results}

Figure 2 summarizes the comparison between the variance of the solar cell output current per month (black lines) and various energetic particle fluxes integrated over a 
month. Here, for the purpose of suppressing irregular rapid components, we take a 4-month smoothing for the variance of the output current per month. The smoothed variance oscillates quasi-periodically with decreasing amplitude. The quasi-periodic variation is associated with the orbital variation of the Akebono satellite. The Akebono satellite has an orbit precession in latitude with a period of about 18 months. When its apogee is located at a high latitude, the variance takes a large negative value, indicating fast degradation. The fast degradation occurs twice in a full precession period, since the satellite apogee visits both northern and southern high latitudes in a period.

The top panel presents a comparison with monthly integrated solar proton flux of the GOES satellites in GEO. The colors of the proton flux lines represent the particle energy range as indicated in the figure. The Akebono satellite has silicon solar cells with a coverglass of $0.5 \mathrm{~mm}$ thick, which gives a penetration range for normal incidence of about 8 MeV protons (e.g., Knoll, 1989). Therefore, proton flux integrated over an energy larger than $10 \mathrm{MeV}$ may represent the particles that damage the solar cells. Heavy ions such as alpha particles cause more degradation, but we assume that heavy ions are included as a portion of the proton flux in this study.

The solar proton flux varies rather randomly whereas the degradation is quasi-periodic. We found no obvious correlation. For a precise comparison, we have to calculate the solar proton flux at the Akebono altitude intruding from GEO. The orbital period of the Akebono satellite was initially 212 min and was much shorter than the typical time scale of solar proton events, so that the effect of satellite location during an orbital motion is expected to be averaged. Therefore, the phase relation of the monthly variation at the Akebono altitude should be similar to that in GEO, though the magnitude of the flux can be different.

Trapped proton flux integrated over a month along the Akebono orbit is shown in the middle panel, which varies periodically regardless of the particle energy range. When the apogee of the Akebono satellite is located at a high latitude, the expected proton flux is large. The Akebono satellite traversed the proton radiation belt centered around $L=1.6$, when its apogee and perigee were located at a high latitude. On the other hand, when its apogee and perigee lay near the equator, the Akebono satellite avoided crossing the center of the proton radiation belt. The apogee $(10,482 \mathrm{~km})$ was too high and the perigee $(272 \mathrm{~km})$ was too low to enter the midst of the proton radiation belt.

The opposite phase relation is found in the trapped electron flux (bottom panel) which also has a quasi-periodic oscillation. When the apogee of the satellite is located at a high latitude, the expected electron flux is low. The contrast with the trapped proton flux results from the presence of the outer belt for trapped electrons. The intervals of large electron flux are not consistent with the fast degradation, and trapped electrons are not thought to be a main cause of the degradation.

Comparing the flux variation among the various space radiations, we find that the intervals of large trapped proton flux coincide fairly well with the fast degradation of the solar cells. The only exception is around the middle of
1991. After 1992, the phase relation was re-established. The results of the comparison with the solar proton and trapped energetic particle flux variations strongly suggest that the oscillating degradation of the Akebono solar cells until 1992 is mainly due to trapped proton radiation, which can be modeled by AP8MAX.

A major interplanetary shock struck the magnetosphere at 03:32 UT on March 24, 1991, leading to a severe geomagnetic storm. CRRES observations revealed that the energetic solar particle event and successive geomagnetic storm drastically changed both the electron and proton radiation belts and the effect lasted, at the least, for more than several months (Blake et al., 1992; Gussenhoven et al., 1996). The proton radiation belt usually has a single peak around $L=1.6$, but a second peak appeared around $L=2 \sim 3$ after the geomagnetic storm. By referring to figure 5 in Gussenhoven et al. (1996), we introduce new models of the proton radiation belt for the interval of inconsistency between the solar cell degradation rate and the proton flux from AP8MAX, which we found in 1991.

Based on the data taken after the magnetic storm in March 1991, Gussenhoven et al. (1996) presented an active time omnidirectional trapped proton flux on the magnetic equator as a function of $L$ for four representative energies, $6,15,26$, and $55 \mathrm{MeV}$. The features of the $L$-shell distribution can be summarized as follows. At low energies, there is a single peak similar to the AP8MAX model profiles, but the shape is less rounded at high $L$ values, falling off more rapidly. At intermediate energies, there is a double-peaked structure lying underneath the AP8MAX profile. At high energies, there is a deep valley between the two peaks. The second peak can be as large as the inner peak, and lies completely above the AP8MAX profile.

Since the CRRES result is highly energy dependent and we rather need the flux integrated over energy, we do not try an exact comparison, but make some models with the basic features described above, seeking any better similarities with the solar cell degradation rate. Inside of an $L$ value of about $1.6 R_{\mathrm{e}}$, there is no significant difference from the AP8MAX profile. But beyond this point, the flux falls more rapidly in some models. The second peak around $L=2.3$ lies beneath, or above, the AP8MAX level in other models.

Figure 3 shows equatorial proton flux distributions with energies higher than $10 \mathrm{MeV}$ (upper panel) and higher than $30 \mathrm{MeV}$ (lower panel) of our new models as a function of the $L$ value. The proton flux provided by AP8MAX is shown by a black line, which yields the monthly-integrated flux used in Fig. 2. The proton flux in our new models is changed along the field lines based on the magnitude of the magnetic field in the same way as in AP8MAX. The second peak of Model 1 and Model 4 is the largest, while that of Model 3 and Model 6 is the smallest. CRRES observation shows that the second peak is more prominent for higher-energy protons. In this respect, Model 3 and Model 6 resemble the $6 \mathrm{MeV}$ and $15 \mathrm{MeV}$ proton distribution of the CRRES observation. Model 2 and Model 4 have an intermediate flux for the second peak, which may be similar to the $26 \mathrm{MeV}$ and $55 \mathrm{MeV}$ proton distribution of the CRRES observation.

We applied the new models from 24 March through to 

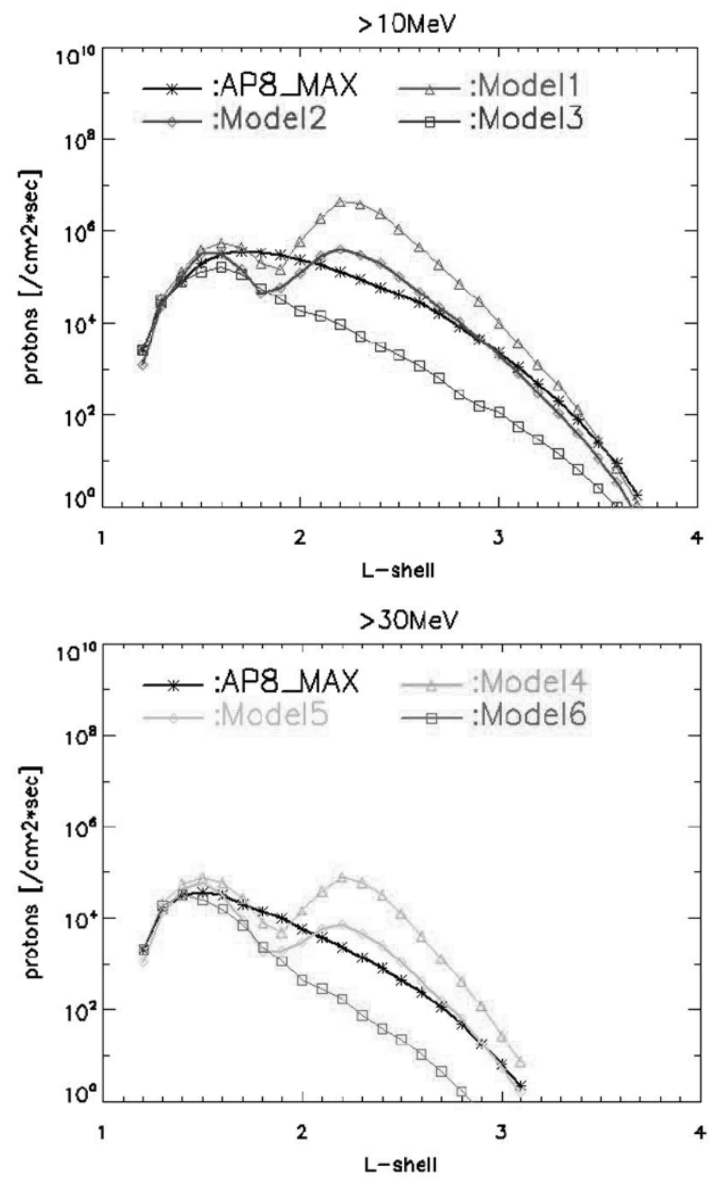

Fig. 3. The equatorial proton flux distribution with energies higher than $10 \mathrm{MeV}$ (upper panel) and higher than $30 \mathrm{MeV}$ (lower panel) of various models as a function of the $L$ value.

the end of November, 1991, to calculate the trapped proton flux integrated over a month along the Akebono orbit. The results are shown in Fig. 4. Model 1 gives the highest flux at the second peak of all the models, including AP8MAX. Model 3 and Model 6 show the lowest flux during the interval. All three models indicate an abrupt large change from the proton flux given by AP8MAX and do not seem suitable to account for the solar cell degradation.

The observed feature of the solar cell degradation rate is that the quasi-periodic variation ceased and the degradation rate stayed rather constant during the interval. Therefore, the proton flux integrated along the satellite orbit should be stable regardless of the phase of the orbit precession in latitude. The proton flux deduced from Model 2 and Model 4 is the most stable among the models. The common feature of the two models is that the second peak is almost equivalent to the first, which provides a rather constant flux during the interval and seems most suitable to account for the observation result.

\section{Discussion}

We found a quasi-periodic degradation rate of the solar cell output current of the Akebono satellite. Comparison of the phase relation with the particle flux variation due to the orbit precession indicates that trapped protons are most responsible for the solar cell degradation of the Akebono
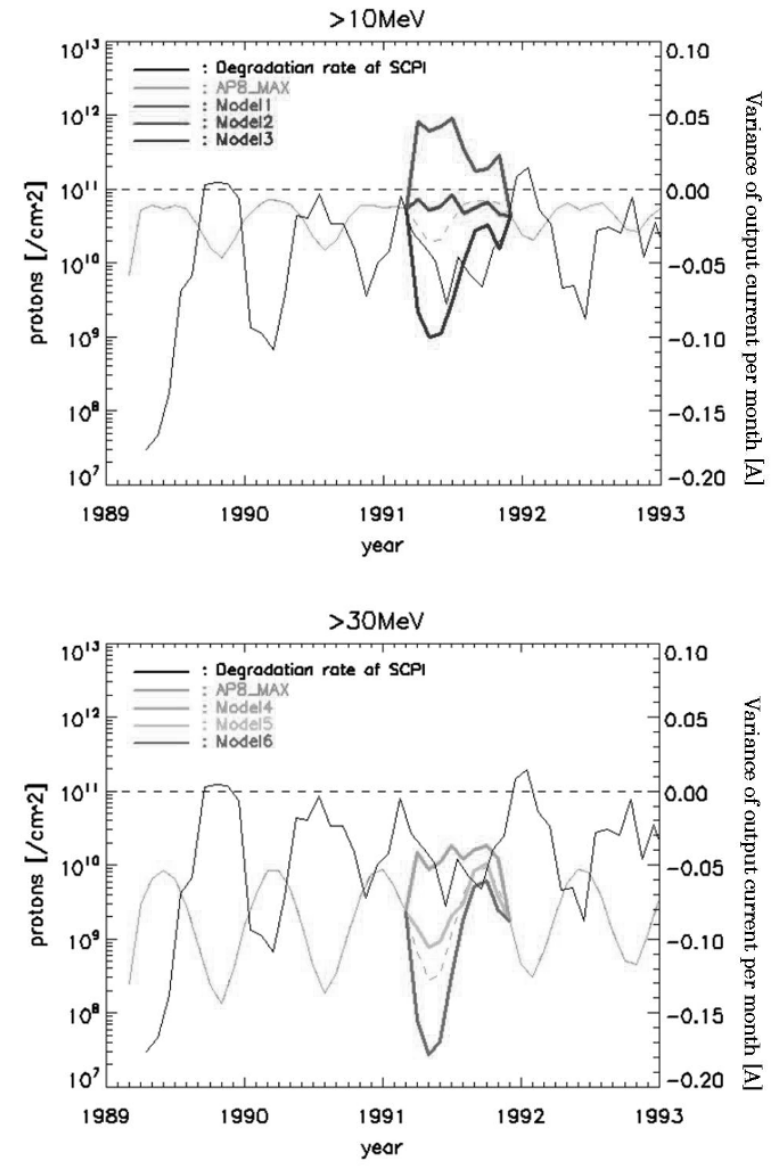

Fig. 4. Variance of the solar cell output current per month and the monthly-integrated trapped proton flux deduced from the various models. The new models are applied from 24 March to the end of November, 1991.

satellite. This is also true for the interval of emergence of the second peak of the proton radiation belt after the intense solar event and following the geomagnetic storm in March 1991. We found a signature of a deformed proton radiation belt in the solar cell degradation rate of the Akebono satellite.

The CRRES observation was terminated in mid-October 1991 and did not show exactly how long the deformed radiation belt remained (Gussenhoven et al., 1996). The SAMPEX satellite was launched in July 1992 and detected a remnant of the deformed electron radiation belt until 1995 (Li and Temerin, 2001). Any similar remnant was not found for the proton radiation belt (e.g., Looper et al., 2005). There is a possibility that the deformation of the proton radiation belt actually lasted near the magnetic equator in 1992, or even in later years, but anisotropic particle distribution could prevent the SAMPEX satellite from detecting energetic protons at low altitude. Our analysis demonstrates that the proton radiation belt really lost its second peak around $L=2.3$, even at high altitude, before the launch of the SAMPEX satellite.

In our models, the proton radiation belt is set to have recovered to the original state rather abruptly at the end of November 1991. A gradual recovery would be more realistic. The duration of about 8 months used in our model 
calculation should be taken as a first-order estimation for the recovery time scale. Our results agree with the fact that the second peak of the proton radiation belt around $L=2 \sim 3$ disappeared after the CRESS observation was terminated in mid-October 1991 and before the SAMPEX satellite started observation in mid-1992.

The Akebono satellite carried a radiation monitor instrument, RDM, on board, and measured energetic electrons and protons. Obara and Li (2003) analyzed the RDM data and studied the rapid formation of a new electron radiation belt during the 1991 event. From the RDM observation, Takagi et al. (1995) also reported a drastic increase around $L=2 \sim 3$ from the proton radiation monitor for the solar proton event and the geomagnetic storm in March 1991. However, its proton measurement suffered from contamination by energetic electron flux and, therefore, we do not attempt a quantitative comparison with our results here. We simply point out that the RDM on board the Akebono satellite also observed a drastic change in the radiation belt which, at least, lasted for more than several months in 1991.

We obtain a fair agreement between the degradation rate and the proton flux in the case of Model 2 for $>10 \mathrm{MeV}$ and Model 4 for $>30 \mathrm{MeV}$ protons. Both cases have a second peak equivalent to the first peak and resemble the $26 \mathrm{MeV}$ and $55 \mathrm{MeV}$ proton distribution of the CRRES observation. Protons with energies of $8 \mathrm{MeV}$ have a penetration range of about $0.5 \mathrm{~mm}$, the thickness of the coverglass of the Akebono satellite. Model 3 and Model 6 have a similar distribution of $15 \mathrm{MeV}$ protons of the CRRES observations, but give a poor agreement. The $L$-shell distribution of Model 2 and Model 4 may seem to represent protons with rather high energies, considering the corresponding penetration range. Protons probably need more energy in order to damage the silicon solar cells even after their passage through the coverglass.

Our result shows that solar protons have not decreased the solar cell output of the Akebono satellite, but solar protons are generally known to be a major cause of solar cell degradation. As an example, the Japanese CS satellite on GEO experienced a step-like decrease of solar cell output for occasions of large solar proton events. A large decrease took place in October 1989, when the Akebono satellite has been also in orbit. We investigated the solar cell output variation of the Akebono satellite in October 1989, but found no evident decrease. Probably due to the low altitude of Akebono orbit, the solar cells are not significantly affected by solar protons but are more severely damaged by trapped proton radiation.

We have analyzed the data of the solar cell output current between 1989 and 1992 in this study. We still have the Akebono data up to the present. From SAMPEX observation,
Looper et al. (2005) reported that the proton radiation belt showed another drastic change in 2003. Although we need to identify and remove other variation components carefully from the solar cell output current, it may be possible to expand our analysis to later years to look for other variations of the proton radiation belt.

Acknowledgments. Successful data acquisition of the Akebono satellite for more than 20 years is attributed to the extensive support of all the members of Akebono project team. We also thank Y. Miyoshi and T. Obara for valuable discussion and comments on this work. The GOES data were obtained through SPIDR of the National Geophysical Data Center.

\section{References}

Blake, J. B., W. A. Kolasinski, R. W. Fillius, and E. G. Mullen, Injection of electrons and protons with energies of tens of $\mathrm{MeV}$ into $\mathrm{L}<3$ on 24 March 1991, Geophys. Res. Lett., 19, 821-824, 1992.

Gussenhoven, M. S., E. G. Mullen, and D. H. Brautigam, Improved understanding of the Earth's radiation belts from the CRRES satellite, IEEE Trans. Nuclear Sci., 43, 353-368, 1996.

Hudson, M. K., A. D. Kotelnikov, X. Li, I. Roth, M. Temerin, J. Wygant, J. B. Blake, and M. S. Gussenhoven, Similation of proton radiation belt formation during the March 24, 1991 SSC, Geophys. Res. Lett., 22, 291294, 1995.

Hudson, M. K., S. R. Elkington, J. G. Lyon, V. A. Marchenko, I. Roth, M. Temerein, J. B. Blake, M. S. Gussenhoven, and J. R. Wygant, Simulation of radiation belt formation during storm sudden commencements, $J$. Geophys. Res., 102, 14087-14102, 1997.

Knoll, G. F., Radiation Detection and Measurement, John Wiley \& Sons, Inc., 1989.

Li, X. and M. A. Temerin, The electron radiation belt, Space Sci. Rev., 95, 569-580, 2001.

Li, X., M. Roth, M. Temerin, J. R. Wygant, M. K. Hudson, and J. B. Blake, Simulation of the prompt energization and transport of radiation belt particles during the March 24, 1991 SSC, Geophys. Res. Lett., 20, 2423-2426, 1993.

Looper, M. D., J. B. Blake, R. A. Mewaldt, J. R. Cummings, and D. N. Baker, Observations of the remnants of the ultrarelativistic electrons injected by the strong SSC of 24 March 1991, Geophys. Res. Lett., 21, 2079-2082, 1994.

Looper, M. D., J. B. Blake, and R. A. Mewaldt, Response of the inner radiation belt to the violent Sun-Earth connection events of October-November 2003, Geophys. Res. Lett., 32, L03S06, doi:10.1029/204GL021502, 2005.

Obara, T. and X. Li, Formation of new electron radiation belt during magnetospheric compression event, Adv. Space Res., 31, 1027-1032, 2003.

Oya, H. and K. Tsuruda, Introduction to the Akebono (EXOS-D) satellite observations, J. Geomag. Geoelectr., 42, 367-370, 1990.

Patel, M. R., Spacecraft Power System, CRC Press, 2005.

Takagi, S., T. Nakamura, T. Kohno, and F. Makino, Flux distribution and modulation of charged particles in the radiation belt observed with Akebono, Bull. ISAS, 80, 1995.

Tsuruda, K. and H. Oya, Introduction to the Exos-D (Akebono) project, Geophys. Res. Lett., 18, 293-295, 1991.

H. Ishikawa, W. Miyake (e-mail: wmiyake@keyaki.cc.u-tokai.ac.jp), and A. Matsuoka 